\title{
Automated Regression Testing for Web Services
}

\author{
Divya Rohatgi, Gyanendra Dwivedi, Tulika Pandey
}

\begin{abstract}
Web services represent class of applications developed through open internet standards. They help to develop dynamic applications and thus are used to deliver business components or functionalities on the web. Business functionalities as per changing market scenarios are frequently changing and so are these web services. It is therefore the need to properly ensure quality of these web services as they are essential to deliver functions which are required for organizations in their strategic business. To maintain desired level of quality, software maintainability of such applications is important. Regression Testing is required for proper maintaining any software and it becomes very costly in terms of cost and labor for constantly changing applications. Thus to reduce maintenance cost we need to reduce effort required in such testing. This paper presents an efficient regression testing strategy which is also automated to reduce the regression costs of web services. The strategy is implemented through an extensive framework supported by automation and also integrates data testing with regression. The proposed work is particularly useful for those applications which are delivered over web.
\end{abstract}

Keywords: Automated testing, Regression Testing, Web based applications, Web Service

\section{INTRODUCTION}

The Web Services represent a cutting edge phenomenon that can provide the advantage to organisations for developing applications and find enormous gains in terms of productivity and efficiency. Instead of the companies to deliver static content or functionality, web services help them to develop and deliver dynamic applications that can be called as per need and can be used in integration with other applications to deliver a whole new functionality. In addition, as per changing market conditions when business conditions are also evolving then developers can change these services as per requirements very easily. It is through these web services that we can modify and adapt applications so efficiently which were not possible earlier with conventional method of software development. Since these services are constantly evolving, so proper regression testing has to be carried out. Regression testing costs increases if the software is constantly evolving. In such scenarios manual selection of test cases becomes very tedious in terms of cost, effort and

Revised Manuscript Received on December 30, 2019.

* Correspondence Author

Divya Rohatgi*, Research Scholar, Department of CS \& IT, SHUATS, Prayagraj, India.

Gyanendra Dwivedi, Ugam Solutions Pvt. Ltd., Mumbai, India.

Dr. Tulika Pandey, Department of CS \& IT, SHUATS, Prayagraj, India.

(C) The Authors. Published by Blue Eyes Intelligence Engineering and Sciences Publication (BEIESP). This is an open access article under the CC BY-NC-ND license (http://creativecommons.org/licenses/by-nc-nd/4.0/) time. Automation of regression test cases is the better option in this situation. Regression Testing is a software maintenance activity to ensure that a recent program or code change has not impacted or changed the already correct features. It is either performed by full selection, partial selection or prioritization of test suite. How to select regression testing technique is the most important activity which can be evaluated by finding maximum errors in a shortest time or cost. Regression Testing can be carried out either by selecting the impacted test cases thereby reducing the test suite or in some cases we can attach some priority to the test cases based on criticality of the module, functionality of the module or business costs. The priority of these test cases decides the order in which the test cases will be executed by the testing team. The decision is taken depending upon the resources, time and cost of running the test cases. For small applications which are frozen as per requirements of the customer, regression testing is easy. Test selection and execution can be performed manually. But for large and complex applications where no of test cases become enormous it is advisable to follow automated approach to save time and effort. For application which are web based particularly web services the scenario becomes all the more challenging due to the dynamic and heterogeneous nature of such services. There are various approaches for regression testing for different types of software. It is also observed that a substantial amount of man-hours are spent by testing team for the regression testing which includes activities like setting up test runs, monitoring cycles of test execution, analysing results, and maintaining testing related data and resources. So it becomes very necessary to decrease efforts required for regression testing if we want to reduce software maintenance cost. There are various automated tools present for different types of testing particularly regression testing in industry. These tools are either free or licensed. So depending upon the requirements and budget of the organisations, the choice of an appropriate tool can be done.

This paper describes an automated regression testing strategy for those applications which are web based particularly web services. The rest of the paper is outlined as follows: Section II presents review of literature detailing the approaches for regression testing. Section III presents the proposed methodology. Results are given in Section IV and Section V summarizes the conclusion.

\section{LITERATURE REVIEW}

Regression testing whether it is manual or automated is a well-researched area. This section presents an overview of review literature for regression testing particularly Web Services. 
Hua Zhong et al. in [1] proposed TestSage which can conduct regression test selection applicable for web services used in big commercial applications. Through little effort the tool TestSage can find out dependencies based on functionality between test case and the web service which has to be tested. Anneliese Andrews et al. [2] proposed a black box approach, selective in nature which is a model based testing for web applications. Singal Prerna et al. [3] demonstrated that those applications which are using web services, the required test cases for regression test can be chosen based on specification changes in Web Services Description Language or in short WSDL. For this, XML file for WSDL is parsed to get required data for name of the operation and input/output messages. Sahoo Sobhana et al. [4] implemented a model which uses change detection algorithm to find out the changes in operation tree which is built for original and changed WSDL. After that, the authors used a forward slicing algorithm which finds out only the impacted and changed parts of the service under consideration. Buck Paul et al. [5] outlines a selective approach for test selection of composite services. Priority based testing is used for such applications. Zarrad Anis et al. [6] produced an organized and detailed review for regression testing strategies. Caturvedi Animesh et al. [7] presented a Supported Approach to Testing the Active Service of Web Services. Authors presented a tool to test web service regressions for both functional and nonfunctional requirements using WSDL files. Chen L. et al. [8] proposed prioritization of test cases used in regression testing. Authors presented a dependence analysis based test case prioritization technique for Web Service regression testing. In [9] Mohanty et al. implemented CFG through which we can choose all those test cases that can find out the changes in SOA applications. The outlined method of selection is shown to be safe. Sahar Tahviliab et al. [10] proposed a new tool ESPRET which can be used to find out the time required to run the test cases manually. For critical systems where safety is an important concern, manual way of testing is also considered effective and important. A. Shi et al. [11] proposed that a greedy algorithm that takes into account dependencies of test cases and historical build data. M. Vasic et al. [12] proposed regression testing by skipping those tests that are not affected by changes in applications. This technique is better as now Agile approach of software development is used and it becomes difficult for testers to perform regression testing with many and frequent changes. Israr Ghani et al. [13] proposed that there is a dramatic increase in popularity of web services because of which there is a need to concentrate on their testing in order to have desired quality. The authors emphasized on quality on service factors like reusability, interoperability, accuracy etc. if we want to have better quality which can be delivered in service. Shunhui Ji et al. [14] presented an approach for BPEL (Business Process Execution Language) composite service. These services are evolving continuously so regression effort increases in such services. R. K. Saha et al. [15] proposed REPiR for prioritization of regression test cases which can actually be seen as Information Retrieval problem. Athira et al. [16] proposed a UML based approach in which activity diagrams are taken into account for describing the system and getting information regarding test coverage. Gothenburg [17] explained that if we select regression test selection strategies at lower and finer level of granularity then it will be more precise in choosing test cases.

\section{PROPOSED REGRESSION TESTING MODEL}

Regression testing for web based applications is an important concern. There are many types of web applications, some are having web services embedded and some are not having. But irrespective of any application which is web based, automation is a smart choice. This section covers the proposed strategy for regression testing which also offers the data testing in addition with functional testing. The strategy is validated on a sample reporting software application available online which are used to take customer feedback or other input as a part of online survey or polls. These reporting software applications are frequently used to take startegic decisions. The user fill the survey options given in an online survey application and then these answers are saving to the database.Then reporting tools are used to get the user input data in reoprt format on basis of various dimensions like geographical location, age wise, gender wise and other filter criteria. They are particularly useful to business managers and data scientists to analyze and draw strategic inferences based on survey or poll submitted by public. Since database access is not given to all so reporting tools are widely used. These reporting applications are inherently web based applications and are prone to frequent changes as per business requirements. So regression testng is important. Also we need to be sure that results which we are getting through database is same as results which we get from these reoprint applications. So data testing is also important with regresssion testing. This approach integrates both regression with data testing and thus gives the added advantage. The approach is integrated in a framework which is automated. Fig. 1 shows the framework for the proposed approach.

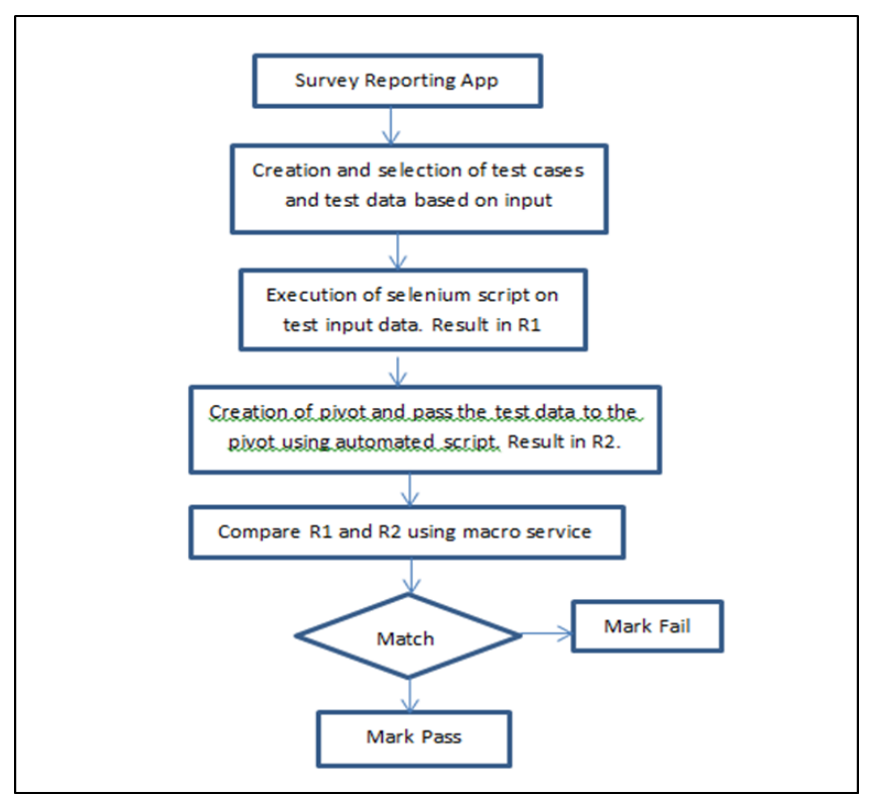

Fig. 1. Proposed Framework

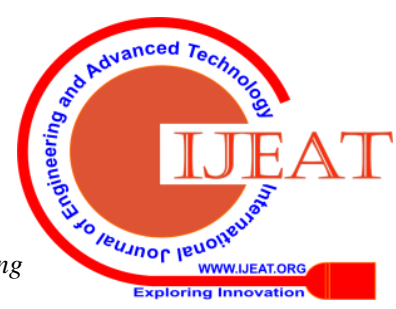


The framework is implemented in the following process :

1. Data is kept in database in transactional form. First test cases of web pages of the reporting application was written. On the basis of that, test data was developed. This test data was kept in some external file. This test data may contain multiple test cases. Fig. 2 shows the sample test case with test data.

\begin{tabular}{|c|c|c|c|c|c|}
\hline A & B & c & 0 & $\varepsilon$ & $\mathrm{f}$ \\
\hline$\#$ & Module & Description & test input date & Expected & |Actual result \\
\hline 1 & test question input & thatch with web application questions & input"1 & Pass & \\
\hline 2 & Question number & $\begin{array}{l}\text { Question sequencing with expected question on } \\
\text { basis of input }\end{array}$ & "input"o & Pass & \\
\hline 3 & navigation Path & $\begin{array}{l}\text { On basis of filter criteria of options question } \\
\text { should be navigate on respective branches of } \\
\text { navigation path }\end{array}$ & linput's & Pass & \\
\hline 4 & Exitcriteria & $\begin{array}{l}\text { Filter criteria on basis of geographical and other } \\
\text { filter criterila }\end{array}$ & inputro & Passs & \\
\hline 5 & $\begin{array}{c}\text { path Coverage of } \\
\text { options }\end{array}$ & inpurto & inputro & Pass & \\
\hline
\end{tabular}

Fig. 2. Sample test case with test data

2. Next in order to reduce test suite, the input is provided to only those test cases where some modification has been made. Modification may be adding, deleting, modifying poll information. Rest of the test cases are rejected. This gives the benefit of regression test selection and which reduces the regression effort.

3. Then a script is created in Selenium which will check the web app using test data kept in the external file. After execution, the report of the regression testing is kept in another file. This is the data from application. Now we need to check the data from database.

4. To do this, simultaneously connection is established from cube to a new file and then pivot is created. Fig. 3 shows sample pivot made.

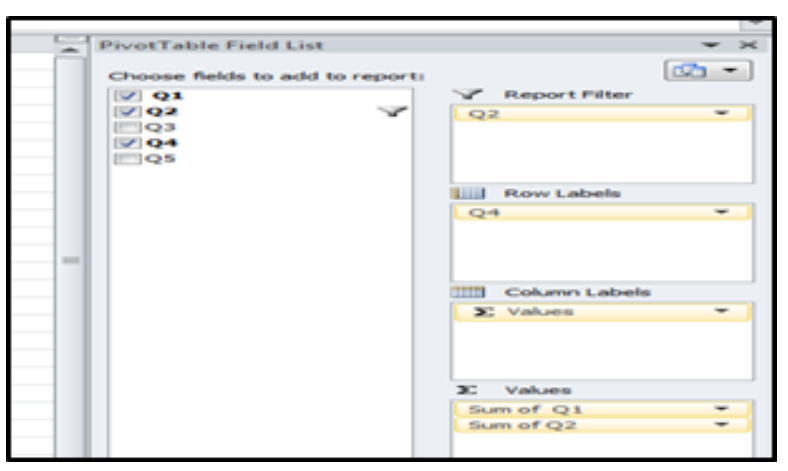

Fig. 3. Sample pivot created

5. Input test data is passed to the pivot and report is stored where we stored the report of data from application.

6. Visual Basic macro service is developed to compare both reports. The basic idea is that since the input to both application and database is same, we should get same result. The macro service will attach Pass/ Fail on depending on the output. Fig. 4 shows the sample VB macro service written.

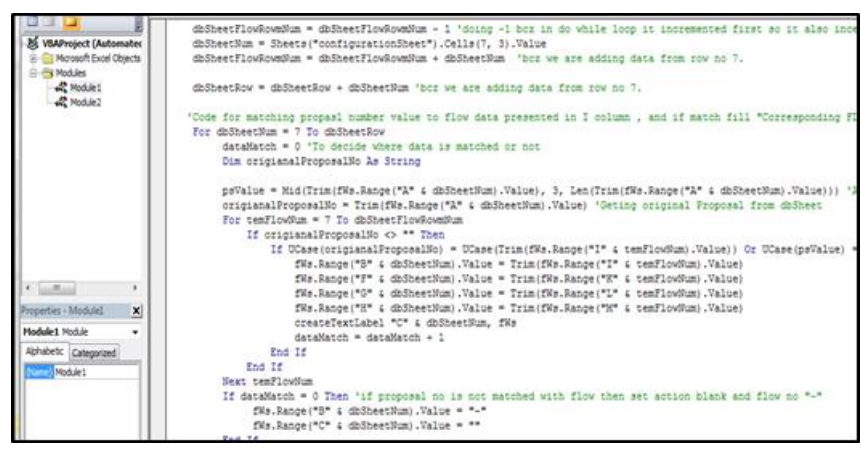

Fig.4. Sample macro service

\section{RESULT AND DISCUSSION}

The results of the implementation of the framework are obtained by passing the developed Visual Basic macro service to compare both reports as shown in Fig. 5. shows the obtained outcome.

\begin{tabular}{|l|l|}
\hline A1_Pass_Test_case_1.2 & A2_Pass_Test_case_2.1 \\
\hline A1_Pass_Test_case_1.3 & A2_Fail_Test_case_2.2 \\
\hline A1_Fail_Test_case_1.7 & A2_Pass_Test_case_2.6 \\
\hline A1_Pass_Test_case_1.9 & A2_Pass_Test_case_2.8 \\
\hline
\end{tabular}

Fig. 5. Result of the execution of the VB Macro Service

In addition, for testing performance of proposed system, a new poll is created using online freely open source applications. The modifications are done in the survey and the reporting application in terms of no of questions and their description. The Table-I below shows the experimental data and Fig. 6 depicts the results graphically

Table-I: Test data

\begin{tabular}{|c|c|c|c|}
\hline $\begin{array}{c}\text { S. } \\
\text { No }\end{array}$ & $\begin{array}{l}\text { No of } \\
\text { test } \\
\text { cases }\end{array}$ & $\begin{array}{c}\text { Test Input } \\
\text { data given } \\
\text { as per } \\
\text { impact of } \\
\text { change }\end{array}$ & $\begin{array}{c}\text { \% } \\
\text { Reduction } \\
\text { in test } \\
\text { suite }\end{array}$ \\
\hline N1 & 10 & 4 & 60 \\
\hline N2 & 10 & 7 & 30 \\
\hline
\end{tabular}

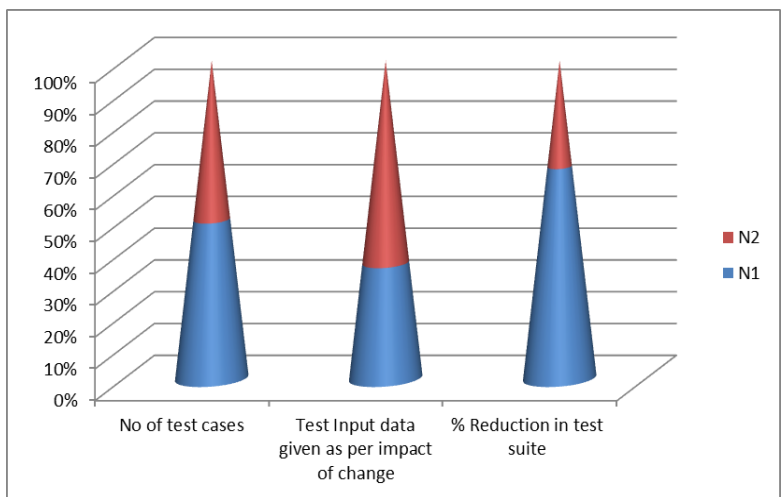

Fig.6. Performance analysis on sample poll

Published By: 
From the results it can be concluded that if number of changes are less, it is better for selection of test input rather than running all test cases. The proposed approach is applicable to web applications having transactional and reporting system. Any application which is web based and available over internet can use this proposed approach. In market there are many methods and approach are available for web applications having web services functional and regression testing. In available software we can either perform the regression testing or function or data testing. In the proposed system we are performing multiple approaches in single framework. The model is architecture neutral and it gives the advantage of quick data processing required for testing. The scope of the presented framework is limited to applications which are web based and which may have a web service embedded. Also such applications should have structured type as a backend database. It reduces the effort for regression testing due to the automation support with the framework which can otherwise be large if done manually forever evolving web based applications.

\section{CONCLUSION}

In this paper an attempt is made to survey regression test approaches for web based applications. Also a novel approach is proposed by which we can reduce effort required in regression testing due to automation support provided. In addition to this it also gives the advantage of data testing particularly for web based reporting systems. As a remark, it can be said that if modifications are few in number then it is advisable to select impacted tests rather than running all test cases. The proposed approach is applicable to web applications having transactional and reporting system. Any application which is web based and available over internet can use this proposed approach.

\section{REFERENCES}

1. Hua Zhong, Lingming Zhang, Sarfraz Khurshid, TestSage: Regression Test Selection for Large-Scale Web Service Testing, 2019 12th IEEE Conference on Software Testing, Validation and Verification (ICST), Xi'an, China, China

2. AnnelieseAndrews, AhmedAlhaddad, SalahBoukhris, Black-box model-based regression testing of fail-safe behavior in web applications, Journal of Systems and Software Volume 149, March 2019, Pages 318-339, Elsevier

3. Prerna Singal ; Anil K Mishra ; Latika Singh, Test case selection for regression testing of applications using web services based on WSDL specification changes, International Conference on Computing, Communication \& Automation,IEEE Xplore, 2015

4. Sobhana Sahoo ; Abhishek Ray, A framework for optimization of regression testing of web services using slicing, 2017 International Conference on Advances in Computing, Communications and Informatics (ICACCI), IEEE Xplore,2017

5. Paul Buck ; Qi Shi ; Áine MacDermott, A Selective Regression Testing Approach for Composite Web Services, 2015 International Conference on Developments of E-Systems Engineering (DeSE), IEEE Xplore

6. Anis Zarrad, A Systematic Review on Regression Testing for Web-Based Applications, Journal of Software 10(8):971-990 August 2015

7. Chaturvedi, Animesh, and Atul Gupta (2013), "A tool supported approach to perform efficient regression testing of web services." Maintenance and Evolution of Service Oriented and Cloud-Based Systems (MESOCA), IEEE 7th International Symposium, pp. 50-55.

8. Chen, L., Wang, Z., Xu, L., Lu, H., \& Xu, B. (2010), "Test case prioritization for web service regression testing", Service Oriented System Engineering (SOSE), Fifth IEEE International Symposium, pp. 173-178.
9. Rajani Kanta Mohanty, Binod Kumar Pattanayak and Durga Prasad Mohapatra, "A Regression Test Selection Technique for SOA Based Applications", International Journal of Software Engineering and Its Applications Vol.8, No.3 (2014), pp.65-72

10. Sahar Tahviliab,Wasif Afzalb, Mehrdad Saadatmanda, Markus Bohlina, Sharvathul Hasan, Ameerjanb, ESPRET: A tool for execution time estimation of manual test cases, Journal of Systems and Software, Volume 146, December 2018, Pages 26-41, Elsevier

11. A. Shi, S. Thummalapenta, S. K. Lahiri, N. Bjorner, and J. Czerwonka. Optimizing test placement for module-level regression testing. In Proceedings of the 39th International Conference on Software Engineering, pages 689-699, 2017

12. M. Vasic, Z. Parvez, A. Milicevic, and M. Gligoric. File-level vs module-level regression test selection for. net. In Joint European Software Engineering Conference and Symposium on the Foundations of Software Engineering, Industry Track, pages 848-853, 2017,ACM,gERMANY

13. Israr Ghani1, Wan M.N. Wan-Kadir2, Ahmad Mustafa3 , Web Service Testing Techniques: A Systematic Literature Review, (IJACSA) International Journal of Advanced Computer Science and Applications, Vol. 10, No. 8, 2019

14. Shunhui Ji ; Bixin Li ; Pengcheng Zhang, Test Case Selection for Data Flow Based Regression Testing of BPEL Composite Services, 2016 IEEE International Conference on Services Computing (SCC)

15. R. K. Saha, L. Zhang, S. Khurshid, and D. E. Perry. An information retrieval approach for regression test prioritization based on program changes. In International Conference on Software Engineering, volume 1, pages 268-279, 2015, ACM

16. Athira, B., \& Samuel, P. (2010), "Web services regression test case prioritization", Proceedings of the International Conference on Computer Information Systems and Industrial Management Applications pp. 438-443.

17. Gothenburg, Sweden , Proceedings of the 40th International Conference on Software Engineering, Gothenburg, Sweden - May 27 - June 03, 2018, Pages 199-209ICSE '18, May 27-June 3, 2018, "Hybrid Regression Test Selection”,ACM

\section{AUTHORS PROFILE}

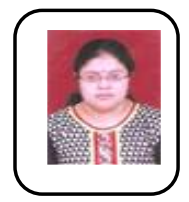

Divya Rohatgi is B.Tech (CSE), M.Tech (CSE) currently and currently is a research scholar in Computer Engineering in dept. of CS\&IT, SHUATS DU, Prayagraj, India. Area of interest includes software testing, data mining and databases.

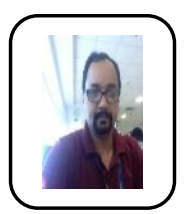

Gyanendra Dwivedi is an alumnus of MNNIT Allahabad and currently working as Manager in Testing \& Quality Assurance in Ugam solutions Pvt. Ltd. Mumbai, India. Area of interest includes software testing and Algorithms.

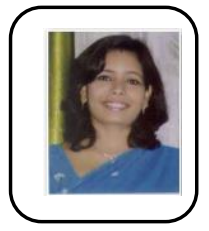

Dr. Tulika Pandey is working as a faculty in Dept. of Computer Science \& Information Technology in Sam Higginbottom University of Agriculture Technology \& Sciences, Prayagraj, UP, India. Area of Interest includes Vehicular Adhoc Network and peer to peer networks. 